\title{
REPRESENTATION THEOREMS ON BANACH FUNCTION SPACES
}

\author{
BY NEIL E. GRETSKY 1 \\ Communicated by F. E. Browder, December 5, 1967
}

Let $L_{\rho}$ be a Banach function space, i.e. a Banach space of (equivalence classes of) measurable point functions on a $\sigma$-finite measure space $(\Omega, \Sigma, \mu)$, with $\rho$ being a function norm possessing at least the weak Fatou property. The results obtained concern integral representations of bounded linear operators from a Banach space $X$ to $L_{\rho}$ and from $L_{\rho}$ (or a subspace) to $X$. These results in some cases complement and in other cases generalize work done in [1], [3], [5], [6], $[7],[12],[13]$.

General notation and results on Banach function spaces can for the most part be found in the first parts of [11]; more detailed work is in [9]. A few further definitions are needed here. If $x$ and $y$ are Banach spaces, let $B(x, y)$ be the space of bounded linear operators from $x$ to $Y$. Distinguish two subrings of $\Sigma$ as $\Sigma_{0}=\left\{E \in \Sigma: \rho\left(\chi_{E}\right)<\infty\right\}$ and $\Sigma_{0}^{\prime}=\left\{E \in \Sigma: \rho^{\prime}\left(\chi_{E}\right)<\infty\right\}$. A partition $\varepsilon$ is defined to be a finite disjoint collection of non- $\mu$-null members of $\Sigma_{0}$ which are of finite measure. The "averaged" step function of a member $f$ of $L_{\rho}$ is defined as

$$
f_{\varepsilon}=\sum_{\varepsilon}\left(\int_{E_{i}}|f| d \mu / \mu\left(E_{i}\right)\right) \chi_{E_{i}} .
$$

A function norm $\rho$ is said to have property $(\mathrm{J})$ if, for each partition $\mathcal{E}, \rho\left(f_{\mathcal{E}}\right) \leqq \rho(f)$. (This is very similar to the levelling property of [5].)

1. The structure of the space $B\left(x, L_{\rho}\right)$.

Definition 1. We define a space of set functions: $V_{\rho}$ $=\left\{x^{*}(\cdot) \mid x^{*}(\cdot): \Sigma_{0}{ }^{\prime} \rightarrow x^{*}, x^{*}(\cdot) x\right.$ is countably additive and $\mu$-continuous for each $x \in X$, and $\left.V_{\rho}\left(x^{*}(\cdot)\right)<\infty\right\}$ where

$$
V_{\rho}\left(x^{*}(\cdot)\right)=\sup _{\|x\| 1} \sup _{\varepsilon} \rho\left(\sum_{\varepsilon} \frac{x^{*}\left(E_{i}\right) x}{\mu\left(E_{i}\right)} \chi_{E i}\right) .
$$

The representation of bounded linear operators from $X$ to $L_{\rho}$ is made in terms of this space.

1 The results announced here are contained in the author's doctoral dissertation written at Carnegie Institute of Technology under the guidance of Professor M. M. Rao. 
THEOREM 1. If $\rho^{\prime}$ possesses property $(\mathrm{J})$, then there is an isomorphism between $B\left(x, L_{\rho}\right)$ and $v_{\rho}$; moreover,

$$
\gamma\|T\| \leqq V_{\rho}\left(x^{*}(\cdot)\right) \leqq \gamma^{-1}\|T\|
$$

for corresponding elements.

(The constant $\gamma$ is fixed for each $L_{\rho}$ space and has value $0<\gamma \leqq 1$ with $\gamma=1$ if and only if $\rho$ has the strong Fatou property. It is the constant which appears in Theorems 1.1 and 1.2 of [11].)

The correspondence is obtained in one direction by defining $x^{*}(E) x=\int_{E} T x(\omega) d \mu(\omega)$ for $E \in \Sigma_{0}^{\prime}$. In the other direction, a type of Radon-Nikodym derivative is used.

2. The structure of the space $B\left(M_{\rho}, \mathfrak{X}\right)$. Results for $B\left(L_{\rho}, \mathfrak{X}\right)$ unfortunately seem not to be, in general, available by the present techniques. Results which will be presented in $\$ 3$ have been obtained for the linear functionals $(x=$ scalars). In the case of a general $x$, we have results for a closed subspace of $L_{\rho}$ (which in some common cases is all of $L_{\rho}$ ).

Definition 2. Let $M_{\rho}=\operatorname{cl}\left\{f \in L_{\rho}: f\right.$ is bounded and has support in $\left.\Sigma_{0}\right\}$.

Note that $M_{\rho}$ is a closed subspace which is normal and a sublattice (in fact, a lattice ideal).

Definition 3. We define a space of set functions: ${ }^{W_{\rho}^{\prime}}=\{x(\cdot) \mid x(\cdot)$ : $\Sigma_{0} \rightarrow X, x(\cdot)$ is finitely additive, vanishes on $\mu$-null sets, and $\left.W_{\rho}^{\prime}(x(\cdot))<\infty\right\}$, where

$$
W_{\rho}^{\prime}(x(\cdot))=\sup _{\left\|x^{*}\right\|<1} \sup _{\varepsilon} \rho^{\prime}\left(\sum_{\varepsilon} \frac{x^{*} x\left(E_{i}\right)}{\mu\left(E_{i}\right)} \chi_{E_{i}}\right) .
$$

To represent elements of $B\left(M_{\rho}, \mathscr{C}\right)$ it will be desirable to integrate members of $M_{\rho}$ against set functions in $W_{\rho}^{\prime}$. In order to do this, Bartle's treatment [2] of integration will be used.

Definition 4. A measurable function $f$ is integrable over $\Omega$ with respect to an $x$-valued finitely additive set function $x(\cdot)$ if there is a sequence $\left\{f_{n}\right\}$ of simple functions such that

(i) $f_{n} \rightarrow f$ in $x(\cdot)$ measure,

(ii) $\lambda_{n}(\cdot)$ are uniformly absolutely continuous, and

(iii) $\lambda_{n}(\cdot)$ are equicontinuous,

where $\lambda_{n}(E)=\int_{E f_{n}} d x$ for $E \in \Sigma$ and where (i), (ii), and (iii) are with respect to the semivariation of $x(\cdot)$.

The fact that if $f \in M_{\rho}$ and $x(\cdot) \in w_{\rho}^{\prime}$ then $f$ is $x(\cdot)$ integrable leads to the representation theorem: 
THEOREM 2. If $\rho^{\prime}$ has $(\mathrm{J})$, then $B\left(M_{p}, X\right)$ and ' $W_{\rho}^{\prime}$ are isomorphic; moreover, $\|T\| \leqq W_{\rho}^{\prime}(x(\cdot)) \leqq \gamma^{-1}\|T\|$ for corresponding elements.

The correspondence is given by $T f=\int f d x$ and $x(E)=T\left(\chi_{E}\right)$ for $E \in \Sigma_{0}$.

COROLLARY. If every member of $M_{\rho}$ has absolutely continuous norm, then each $x(\cdot) \in W_{\rho}^{\prime}$ is $\mu$-continuous.

3. Representation of linear functionals. We have obtained two characterizations of $L^{*}$. One assumes property $(\mathrm{J})$, the other does not. Both results proceed by use of the quotient space $\left(L_{\rho} / M_{\rho}\right)^{*}$. Define $N_{\rho}=L_{\rho} / M_{\rho}$ and equip $N_{\rho}$ with the usual factor norm and order (recalling that $M_{\rho}$ is a lattice ideal). Denote the canonical map as $\lambda: L_{\rho} \rightarrow N_{\rho}$. Note that $\lambda$ is continuous, interior, homomorphic (both linear and lattice), and has norm $\leqq 1$. In addition $\lambda^{*}: N_{\rho} \rightarrow M_{\rho}$ is an isometric isomorphic surjection. (Note that $N_{\rho}$ is an $A B$ lattice, even though it is not a Banach function space over the given measure space.) In $L_{\rho}$ we define the convex, norm-determining and (in general) nonlinear subset $\widetilde{L}_{\rho}=\left\{f: f=\bigvee_{i=1}^{n} f_{i}, f_{i} \geqq 0, \rho\left(f_{i}\right) \leqq 1,1 \leqq n<\infty\right\}$. Since $\lambda$ is interior, $\lambda\left(\tilde{L}_{\rho}\right)$ contains the nonnegative elements of the open unit ball of $N_{\rho}$. It is here that an assumption is needed:

Condition (I). $\lambda\left(\tilde{L}_{\rho}\right)$ lies in the closed unit ball of $N_{\rho}$. (With this assumption, $N_{\rho}$ is an AL space in the sense of Kakutani.)

The characterization of $N_{\rho}^{*}$ is in terms of certain additive set functions. Define ba $(\Omega, \Sigma, \mu)$ to be the collection of bounded additive set functions on $\Sigma$ which vanish on $\mu$-null sets. Denote by ca $(\Omega, \Sigma, \mu)$ the countably additive members of ba $(\Omega, \Sigma, \mu)$ and by pfa $(\Omega, \Sigma, \mu)$ the purely finitely additive members of $\mathrm{ba}(\Omega, \Sigma, \mu)$. We will need to integrate elements of $N_{\rho}$ with respect to set functions in $\mathrm{pfa}(\Omega, \Sigma, \mu)$. The integration used is a variant of that found in [14] and [15].

Definition 5. Let $0 \leqq \nu \in \operatorname{pfa}(\Omega, \Sigma, \mu)$ and $0 \leqq f \in L_{\rho}$. Define $I_{\nu}(f)$ $=\inf \left\{\sum_{i=1}^{n}\left\|\lambda\left(f \chi_{E_{i}}\right)\right\| \nu\left(E_{i}\right):\left\{E_{i}\right\}\right.$ disjoint finite partition of $\left.\Omega\right\}$.

This has all the usually desired properties of an integral and is extended to all of $L_{\rho}$ and $\mathrm{pfa}(\Omega, \Sigma, \mu)$ by linearity on the decomposition into their positive parts. Note that one could equally well write $I_{\nu}(\lambda(f))$ since $I_{\nu}$ is constant over cosets.

THEOREM 3. Assuming that condition (I) holds, there is an isometric isomorphism which is also a lattice isomorphism between $N_{p}^{*}$ and a closed subspace of $\operatorname{pfa}(\Omega, \Sigma, \mu)$ which shall be denoted as $\mathcal{P}_{p^{\prime}}$. The isometry is $\left\|z^{*}\right\|=|\nu|(\Omega)$.

The correspondence is given by: for $\nu \in \mathcal{P}_{\rho^{\prime}}, z^{*}(\lambda(f))=I_{\nu}(f)$, $f \in L_{p} ;$ for $0 \leqq z^{*} \in N_{p}^{*}, \nu(E)=\left\|z_{E}^{*}\right\|, E \in \Sigma$; and for general $z^{*} \in N_{p}^{*}$ one 
uses its decomposition into positive parts. (We denote $z_{E}^{*}(\lambda(f)$ ) $=z^{*}\left(\lambda(f) \chi_{E}\right)$.)

The space $\mathcal{P}_{\rho^{\prime}}$ is determined as the range of the (bounded) projection obtained by composing the correspondence from $\operatorname{pfa}(\Omega, \Sigma, \mu)$ into $N_{\rho}^{*}$ with that from $N_{\rho}^{*}$ to $\operatorname{pfa}(\Omega, \Sigma, \mu)$. One may describe $\rho_{\rho^{\prime}}$ as those elements in $\operatorname{pfa}(\Omega, \Sigma, \mu)$ whose support lies inside the support of a function in $\tilde{L}_{\rho}$ which is not in $M_{\rho}$.

TheOREM 4. The conjugate space $L_{\rho}^{*}$ has a direct sum decomposition into two closed linear (lattice) subspaces which are seminormal, namely into $M_{\rho}^{\perp}$ and its lattice orthogonal complement $\left(M_{\rho}^{\perp}\right)^{0.0}$ which is isometrically linearly and lattice isomorphic to $M_{\rho}^{*}$. Moreover, in the decomposition, $\left\|x^{*}\right\|=\left\|y^{*}\right\|+\left\|z^{*}\right\|$ where $x^{*}=y^{*}+z^{*}$ with $y^{*} \in M_{\rho}^{\perp}$ and $z^{*} \in\left(M_{\rho}^{\perp}\right)^{0.0}$.

Thus in order to represent $L_{\rho}^{*}$ all that is needed is a representation of $M_{\rho}^{*}$. If $\rho^{\prime}$ has property $(\mathrm{J})$, then by Theorem $2, M_{\rho}^{*}$ is isomorphic to the space of set functions $W_{\rho^{\prime}}^{R}=\left\{G \mid G: \Sigma_{0} \rightarrow\right.$ reals, $G$ finitely additive on $\Sigma_{0}, G$ vanishes on $\mu$-null sets, and $W_{\rho^{\prime}}(G)$ $\left.=\sup _{8} \rho^{\prime}\left(\sum_{\varepsilon}\left(G\left(E_{i}\right) / \mu\left(E_{i}\right)\right) \chi_{E_{i}}\right)<\infty\right\}$ under the correspondence $G(E)=x^{*}\left(\chi_{E}\right)$ for $E \in \Sigma_{0}$, and $x^{*}(f)=\int f d G$ for $f \in M_{\rho}$. For corresponding elements, one has $\left\|x^{*}\right\| \leqq W_{\rho^{\prime}}(G) \leqq \gamma^{-1}\left\|x^{*}\right\|$. (It is also true that this correspondence is a lattice isomorphism.)

If we define $a_{\rho^{\prime}}=w_{\rho^{\prime}}^{R} \times \rho_{\rho^{\prime}}$ with norm $\|(G, \nu)\|=W_{\rho^{\prime}}(G)+|\nu|(\Omega)$ and with partial order $(G, \nu) \geqq(0,0)$ if $G \geqq 0$ and $\nu \geqq 0$, then $Q_{\rho}$, is a Banach lattice and we have:

THEOREM 5. If condition (I) holds and $\rho^{\prime}$ has property (J), then the space $L_{\rho}^{*}$ is linear and lattice isomorphic to $Q_{\rho^{\prime}}$. Moreover the correspondence is a topological equivalence.

There is another characterization of $M_{\rho}^{*}$ that is available without the assumption that $\rho^{\prime}$ has $(\mathrm{J})$. However, this form is not as useful since the norm computation does not explicitly involve the associate space (although if $\rho^{\prime}$ has $(\mathrm{J})$, this approach leads to a norm equivalent to the one given above).

THEOREM 6. There is an isometric isomorphism between $M_{p}^{*}$ and the Banach space $V=\left\{\nu \mid \nu(\cdot)\right.$ real valued, additive set function on $\Sigma_{0}$ which vanishes on $\mu$-null sets, and $\|\nu\|=\sup \left[\left|\int f d \nu\right|: f\right.$ simple and $\left.\rho(f) \leqq 1\right]$ $<\infty\}$. Moreover, the members of $v$ are all countably additive if and only if every function in $M_{\rho}$ is of absolutely continuous norm.

If we define $B_{\rho^{\prime}}$ as $U \times \mathcal{P}_{\rho^{\prime}}$ with norm $\|(\nu, \psi)\|=\|\nu\|+|\psi|(\Omega)$ and 
partial order $(\nu, \psi) \geqq(0,0)$ if $\nu \geqq 0$ and $\psi \geqq 0$, then $\Theta_{p^{\prime}}$ is a Banach lattice and we have:

THEOREM 7. Under the assumption of (I) alone, the space $L_{\rho}^{*}$ is linearly and lattice isomorphic to $B_{p^{\prime}}$. Moreover the correspondence is an isometry.

Details and proofs will appear in Trans. Amer. Math. Soc.

\section{REFERENCES}

1. T. Ando, Linear functionals on Orlicz spaces, Nieuw. Arch. Wisk. (3) 8 (1960), 1-16.

2. R. G. Bartle, A general bilinear vector integral, Studia Math. 15 (1956), 337-352.

3. N. Dunford and B. J. Pettis, Linear operations on summable functions, Trans. Amer. Math. Soc. 47 (1940), 323-392.

4. N. Dunford and J. T. Schwartz, Linear operators, Part I: General theory, Interscience, New York, 1958.

5. H. W. Ellis and I. Halperin, Function spaces determined by a levelling length function, Canad. J. Math. 5 (1953), 576-592.

6. L. F. Kantorovitch and B. Z. Vulich, Sur la representation des operations lineares, Compositio Math. 5(1938), 119-165.

7. G. G. Lorentz and D. G. Wertheim, Representation of linear functionals on Köthe spaces, Canad. J. Math. 5 (1953), 568-575.

8. W. A. J. Luxemburg, Banach function spaces, Ph.D. Thesis, Delft, 1955.

9. W. A. J. Luxemburg and A. C. Zaanen, ${ }^{2}$ Notes on Banach function spaces, Nederl. Akad. Wetensch. Proc. Ser A = Indag. Math., Note I, 66 (1963), 135-147; Note II, 66 (1963), 148-153; Note III, 66 (1963), 239-250; Note IV, 66 (1963), 251263; Note V, 66 (1953), 496-504; Note VI, 66 (1953), 655-668; Note VII, 66 (1953), 669-681; Note VIII, 67 (1964), 104-119; Note IX, 67 (1964), 360-376; Note X, 67 (1964), 493-506; Note XI, 67 (1964), 507-518; Note XII, 67 (1964), 519-529; Note XIII, 67 (1964), 530-543; Note XIV, 68 (1965), 229-248; Note XV, 68 (1965), 415-446; Note XVI, 68 (1965), 646-667.

10. - Some remarks on Banach function spaces, Nederl. Akad. Wetensch. Proc. Ser A = Indag. Math. 59 (1956), 110-119.

11. - Compactness of integral operators in Banach function spaces, Math. Ann. 149 (1963), 150-180.

12. M. M. Rao, Linear functionals on Orlicz spaces, Nieuw. Arch. Wisk. 12 (1964), 77-98.

13. - Linear functionals on Orlicz spaces: General theory (to appear).

14. P. C. Rosenbloom, Quelques classes de problemes extremaux, Bull. Sci. Math. France 79 (1951), 1-58; 80 (1952), 183-215.

15. A. E. Taylor, Introduction to functional analysis, Wiley, New York, 1958.

16. J. J. Uhl, Orlicz spaces of additive set functions and set-martingales, $\mathrm{Ph} . \mathrm{D}$. thesis, Carnegie Inst. of Tech., 1966.

Carnegie-Mellon University and University of CAlifornia, Riverside

2 Notes XIV, XV, and XVI are by W. A. J. Luxemburg alone. 